\title{
The Role of Mean Platelet Volume as a Predictor of Mortality in Critically Ill Patients: A Systematic Review and Meta-Analysis
}

\author{
Pattraporn Tajarernmuang, ${ }^{1}$ Arintaya Phrommintikul, ${ }^{1}$ Atikun Limsukon, ${ }^{1}$ \\ Chaicharn Pothirat, ${ }^{1}$ and Kaweesak Chittawatanarat ${ }^{2}$ \\ ${ }^{1}$ Department of Internal Medicine, Faculty of Medicine, Chiang Mai University, Chiang Mai 50200, Thailand \\ ${ }^{2}$ Department of Surgery, Faculty of Medicine, Chiang Mai University, Chiang Mai 50200, Thailand \\ Correspondence should be addressed to Kaweesak Chittawatanarat; kchittaw@gmail.com
}

Received 16 November 2015; Accepted 11 January 2016

Academic Editor: Hector Wong

Copyright (C) 2016 Pattraporn Tajarernmuang et al. This is an open access article distributed under the Creative Commons Attribution License, which permits unrestricted use, distribution, and reproduction in any medium, provided the original work is properly cited.

\begin{abstract}
Background. An increase in the mean platelet volume (MPV) has been proposed as a novel prognostic indicator in critically ill patients. Objective. We conducted a systematic review and meta-analysis to determine whether there is an association between MPV and mortality in critically ill patients. Methods. We did electronic search in Medline, Scopus, and Embase up to November 2015. Results. Eleven observational studies, involving 3724 patients, were included. The values of initial MPV in nonsurvivors and survivors were not different, with the mean difference with 95\% confident interval (95\% CI) being 0.17 (95\% CI: $-0.04,0.38 ; p=$ 0.112). However, after small sample studies were excluded in sensitivity analysis, the pooling mean difference of MPV was $0.32(95 \%$ CI: $0.04,0.60 ; p=0.03$ ). In addition, the MPV was observed to be significantly higher in nonsurvivor groups after the third day of admission. On the subgroup analysis, although patient types (sepsis or mixed ICU) and study type (prospective or retrospective study) did not show any significant difference between groups, the difference of MPV was significantly difference on the unit which had mortality up to 30\%. Conclusions. Initial values of MPV might not be used as a prognostic marker of mortality in critically ill patients. Subsequent values of MPV after the 3rd day and the lower mortality rate unit might be useful. However, the heterogeneity between studies is high.
\end{abstract}

\section{Introduction}

Critically ill patients are defined as those patients who have physiologic instability, which may lead to morbidity and mortality within a short period of time. These patients need intensive monitoring of organs functions such as functions related to the cardiovascular, the respiratory, and the neurological systems. Most critically ill patients are transferred to the intensive care unit (ICU) for close monitoring. There are approximately 4 million ICU admissions per year in the United States with average mortality rate reported ranging from $8 \%$ to $19 \%$, or about 500,000 deaths annually, in which sepsis is one of the leading causes of admission and mortality. As we all know, sepsis is an inflammatory response of host to infection. Deterioration of sepsis leads to severe sepsis and septic shock, which has a high mortality rate. Approximately
150,000 patients die annually in Europe and more than 200,000 annually in the United States due to severe sepsis $[1,2]$.

It is not uncommon that the hematologic system is affected by potent inflammation in critically ill patients. The hematologic profiles, including hematocrit, white blood cell count, and platelet count, are used in many widely accepted prognostic prediction scores such as sequential organ failure assessment (SOFA) score, multiple organ dysfunction score (MODS), and logistic organ dysfunction score (LODS) [3-5].

Currently, many physicians have shown interest in platelet indices, particularly the platelet volume, because it may reflect the platelet function better than the platelet count itself. Nowadays, platelet volume is usually reported in routine complete blood count results. The mean platelet volume (MPV) reflects the platelet size. Elevation of MPV is 
suggestive of increasing platelet production and activation. Larger platelets also contain more granules and prothrombotic materials [6, 7]. Many clinical studies have shown an association between high MPV and thromboembolic events, also with adverse cardiovascular and cerebrovascular outcomes. A recent meta-analysis showed the prognostic value of MPV in coronary artery diseases (CAD) [8-11].

However, in non-CAD critical illnesses, the correlation between high MPV and poor prognosis is still unclear. Many cohort studies showed an association between MPV and poor outcome in critically ill patients, but the same was not the case in other studies.

The primary objective of this analysis is to determine the association between MPV and mortality in non-CAD critically ill patients. The secondary objective is to determine the association between MPV and mortality in the subgroup of patients with sepsis.

\section{Materials and Methods}

2.1. Search Strategies. We searched for published and unpublished studies from Medline, Scopus and Embase databases with restrictions in terms of neither the published year nor the language. The MeSH Terms included "mean platelet volume" or "platelet index" or "platelet size" and "sepsis" or "septic shock" or "critically-ill" or "intensive care." The search strategies of each database are described in the Supplemental Appendix A in Supplementary Material available online at http://dx.doi.org/10.1155/2016/4370834. Hand searching was also performed in the reference lists of the included studies to identify additional eligible studies. Additionally, one author (Pattraporn Tajarernmuang) attempted to contact a corresponding author regarding an unpublished study but failed to obtain the data.

2.2. Selection of Studies. All English abstract published studies were selected if they provided the data regarding MPV in intensive care units or sepsis patients and if mortality was the outcome of interest. The included studies needed to have sufficient data for pooling, that is, mean and standard deviation (SD) or median and interquartile range (IQR) of MPV between the groups of surviving and nonsurviving patients and the number of patients in each group. Studies were excluded if there were insufficient data and if there was no response from the authors.

2.3. Data Extraction. The data of eligible studies were independently extracted by two authors (Pattraporn Tajarernmuang and Arintaya Phrommintikul). The baseline characteristics of the included studies were, first, the author's name, publication year, study design, number of patients in survival and nonsurvival groups, age, sex, APACHE II score (if provided), and SOFA score (if provided). The parameters of interest were mean $( \pm S D)$ of MPV and daily MPV in each group (if provided). If results were expressed as median and $\mathrm{IQR}$, the mean $( \pm \mathrm{SD})$ of MPV was acquired by contacting the corresponding authors or by converting using the formula, as suggested by Wan et al. [12]. Disagreements, if any, were resolved after discussion.
2.4. Risk of Bias Assessment. The quality and risk of bias of each study, using the Newcastle and Ottawa risk of bias criteria [13], were assessed independently by two reviewers (Pattraporn Tajarernmuang and Arintaya Phrommintikul). There were three domains to assess, that is, the selection of the study group, the comparability of the groups, and the ascertainment of outcome. The total score was 9; the higher the score, the lower the risk of bias. Disagreements, if any, were discussed, and then the conclusions were made.

2.5. Statistical Analysis. The mean values of MPV between the survival and the nonsurvival groups were estimated and pooled using the unstandardized mean difference (USMD). Heterogeneity between the studies was assessed using $Q$ statistic and $I$ square, which could be obtained from the inclusion and the exclusion criteria of each study, the mortality rate in each study, the level of hospital care, and the method and the time of the MPV measurement. If heterogeneity was present $\left(I^{2}>25 \%\right.$ or $\left.p<0.10\right)$, the Dersimonian and Laird method (random effects models) was applied for all comparisons. Otherwise, if no heterogeneity was observed, the inverse variance method (fixed effects model) was used instead $[14,15]$. Publication bias was examined using the Egger test and the funnel plot [16]. All the statistical analyses were carried out by using the STATA software, version 12. A $p$ value $<0.05$ was considered statistically significant for the two-sided test.

\section{Results}

Three hundred and nineteen publications were identified: 76 from Medline, 89 from Scopus databases, 152 from Embase, and 2 from hand searching (last update on 4 November 2015). A total of 185 publications were duplicated and, thus, were excluded. A total of 188 titles and abstracts were screened and 174 articles were excluded. Fourteen publications were reviewed thoroughly, and 3 of these were excluded due to invalid data. Finally, 11 cohort studies, involving 3724 subjects, were included [17-27]. Reasons for exclusion from the studies are shown in Figure 1. Two of the excluded articles were abstracts from poster presentation, the full publication of which could not be found, and the data from the abstract were not sufficient for pooling [28].

The characteristics of the study subjects are shown in Table 1. Most studies were of cohort type; however, eight of them were retrospective $[17,19-23,25]$. MPV in subsequent days, which was available in five studies, is shown in Supplemental Table 1. The risk of bias assessment of ten eligible studies, using the qualitative assessment form, is presented in Supplemental Table 2. The total scores were found to range from 5 to 8 .

Eight of eleven studies reported using mean MPV and SD of survivors and nonsurvivors in septic patients [17, 18, 20, $21,23,25-27]$. The other three studies reported using median MPV and IQR in each group [19, 22, 24]. After our attempts to establish contact with the authors, we could obtain raw data from one author, from which we could analyze the mean and the SD [24]. For the other two studies, we converted the median and the IQR to mean and SD using the formula 


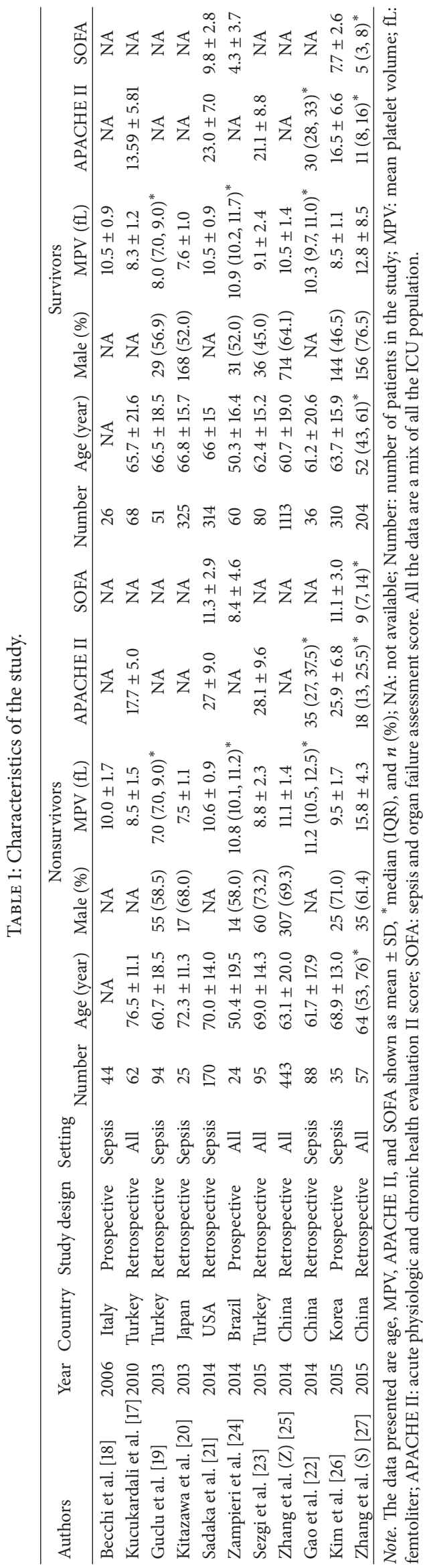




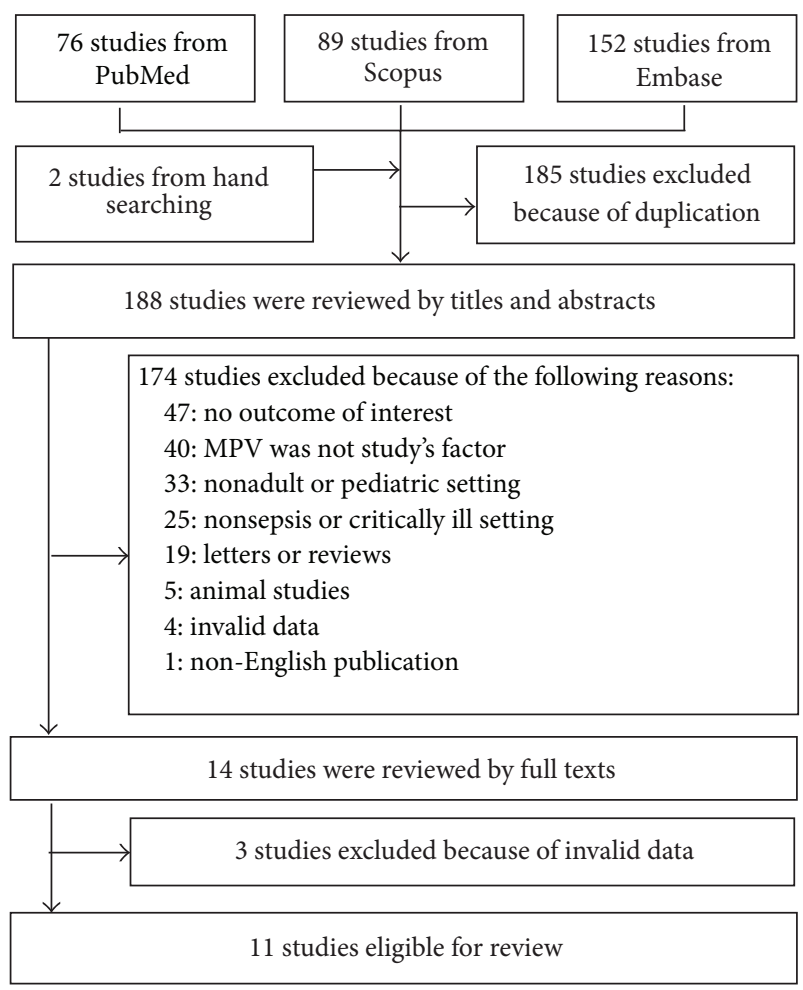

FIGURE 1: The flow chart of the study selection.

suggested by Wan et al. [12]. All the data were pooled and categorized into survival group and nonsurvival group. Six of eleven studies showed higher MPV in the nonsurvival group than in the survival group at the time of enrollment $[18,19$, 22, 24-26]. The pooled mean difference of MPV between nonsurvivors and survivors was 0.17 (95\% CI: $-0.04,0.38$ ); however, there was marked heterogeneity by the random effect model (Chi square $=58.47$, degree of freedom: $10, I^{2}$ : $82.9 \%, p<0.001)$. See Figure 2.

The possible sources of heterogeneity were explored by age, sex, APACHE II, SOFA score study design, ICU setting, and mortality rate using metaregression analysis, and none of those factors was found to be the reason for the heterogeneity (Supplemental Table 3). The subgroup analysis of septic patients also showed no difference between survivors and nonsurvivors, with a mean difference of 0.17 (95\% CI: -0.21 , 0.55 ) (Table 2 and Supplemental Figure 1). However, the heterogeneity of this pooling was also high, as shown in Table 2. Although the tendency of MPV difference between survival and nonsurvival in retrospective study reports was higher than prospective study reports, the heterogeneity of this pooling was high (Table 2 and Supplemental Figure 2). In studies with mortality rate higher than $30 \%$, the mean MPV also tends to be higher in nonsurvivors than in survivors, though not statistically significant (Table 2 and Supplemental Figure 3). In the sensitivity analysis, the heterogeneity studies were excluded by their possible heterogeneity characters and demonstrated in Table 3. Although the heterogeneity was high, the pooling mean of MPV was significantly higher in nonsurvivors after the small sample studies were excluded.
The mean MPV difference was 0.24 (95\% CI: 0.02 to 0.03 ) (Table 3 and Supplemental Figure 4).

The subsequent MPV values were also evaluated. Significant differences in MPV between nonsurvivors and survivors were observed on the third day after admission. The mean MPV differences on the $3 \mathrm{rd}$, 4th, 5th, and 7th days were 0.54 (95\% CI: 0.05, 1.02), 0.64 (95\% CI: 0.38, 0.90), 0.84 (95\% CI: $0.42,1.26$ ), and 0.57 (95\% CI: $0.26,0.88)$, respectively (Table 2 , Figure 3, and Supplemental Figures 5-10).

The funnel plot showed asymmetry, which could be from either heterogeneity of studies or publication bias; interpretation by smaller studies showing statistical significance was not included (Supplemental Figure 11). However, Egger's test did not suggest small study effect (coefficient $=0.50, p$ value $=0.197)$.

\section{Discussion}

To our knowledge, this is the first systematic review and meta-analysis that assesses the prognostic value of MPV in noncardiac critically ill patients. There were eleven studies, including 3724 participants, totally, and the examination was for the association of MPV and mortality in critically ill patients. We found no significant correlation between initial MPV and hospital death. Subgroup analysis by sepsis, ethnicity, and mortality rate did not show any significant difference, as well.

However, we found that the gradual increase in MPV after a few days of admission was associated with increased hospital mortality (Supplemental Table 1). Increasing of MPV on subsequent days after admission in nonsurvivors was reported in five publications $[18,20,22,24,26]$. Two prospective studies demonstrated significant correlation between increased MPV and short-term mortality [24, 26]. Another study showed that the mean MPV level at the time of discharge was higher than the initial MPV level in nonsurvivors, while it was found to be decreasing in the survival group [23]. Therefore, it is evident that sequential monitoring of changes in MPV could be more important than single measurement.

Platelets play an important role in thrombogenesis [29]. The correlation between platelet activation and adverse clinical outcome of vascular diseases including coronary artery disease (CAD), stroke, and venous thromboembolism has been established. The mechanism of alteration of platelet function in sepsis is still unclear. The shape of platelets changes from discoid to spherical with pseudopodia during activation. MPV reflects the average size of platelets. Young platelets are larger than old platelets. Increased number of young platelets indicates increased platelet production due to overconsumption induced by inflammation. Larger platelets are functionally, metabolically, and enzymatically more active than smaller ones. They contain more intracellular thromboxane A2 and increased expression of procoagulant surface proteins such as p-selectin and glycoprotein IIIa, causing greater prothrombotic potential. Moreover, plateletneutrophil interactions and platelet-endothelial interactions facilitate a variety of immune activation instances $[6,7,30$, 31]. 
TABLE 2: Heterogeneity of pool mean differences of MPV in all studies and subgroup of patient type, study type, and mortality rate.

\begin{tabular}{|c|c|c|c|c|c|}
\hline & Number of studies & $I$ square (\%) & SMD & (95\% confidence interval) & $p$ value \\
\hline All studies & 11 & 82.9 & 0.169 & $(-0.040$ to 0.378$)$ & 0.112 \\
\hline \multicolumn{6}{|l|}{ Patient types } \\
\hline Sepsis & 6 & 86.7 & 0.166 & $(-0.215$ to 0.546$)$ & 0.393 \\
\hline All ICU & 5 & 75.9 & 0.169 & $(-0.040$ to 0.378$)$ & 0.160 \\
\hline \multicolumn{6}{|l|}{ Study type } \\
\hline Prospective & 3 & 90.0 & 0.133 & $(-0.663$ to 0.930$)$ & 0.743 \\
\hline Retrospective & 8 & 81.8 & 0.169 & $(-0.040$ to 0.378$)$ & 0.120 \\
\hline \multicolumn{6}{|l|}{ Mortality rate } \\
\hline More than $60 \%$ & 3 & 89.8 & 0.044 & $(-0.692$ to 0.781$)$ & 0.906 \\
\hline $30-60 \%$ & 3 & 1.1 & 0.060 & $(-0.085$ to 0.205$)$ & 0.418 \\
\hline Up to $30 \%$ & 5 & 77.3 & 0.321 & (0.043 to 0.598$)$ & 0.023 \\
\hline
\end{tabular}

SMD: standardized mean difference.

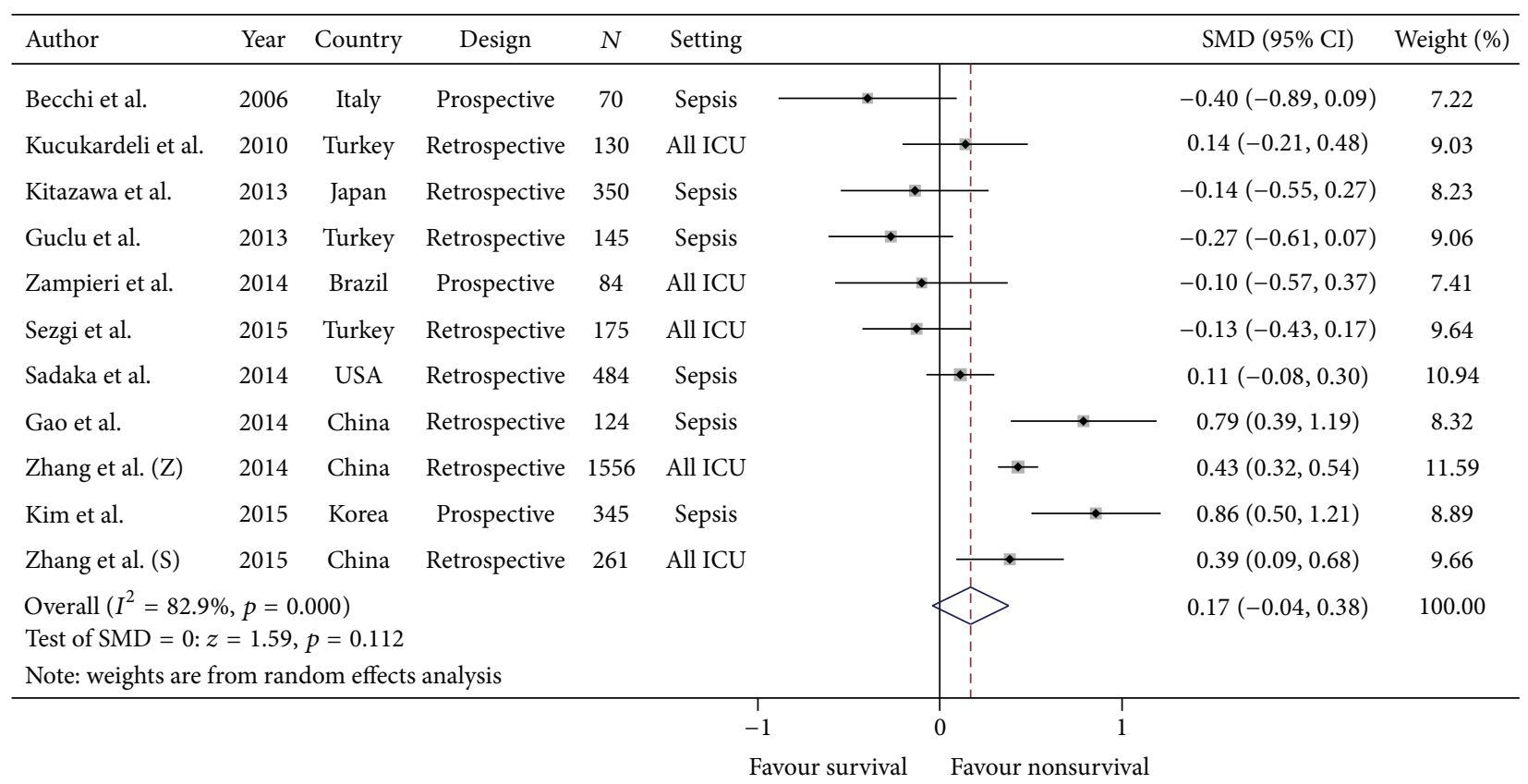

FIGURE 2: The pooled mean differences of the mean platelet volume between critically ill nonsurvivors and survivors.

MPV is available in laboratories worldwide; it is simple and cost-effective enough to be used as one of the prognostic markers in critically ill patients. In 1983, Van der Lelie and Von dem Borne demonstrated the existence of higher MPV in invasive infection than in localized infection and that the MPV value returned to normal when the disease became under control [32].

Our study showed the prognostic potential of the MPV trend to be better in less severe groups than in more severe groups (Table 2). MPV may be a useful predictor of prognosis when the coagulation system and the platelets are still strongly activated in early sepsis but not when coagulation factors and platelets are depleted in late or severe sepsis. Zampieri et al. showed that adding MPV to previous prognostic marker tools such as SAP-3 could increase the prognostic capability [24].
The limitation of our study was that most of the included studies were retrospective. The baseline MPV values of all the studies were different; consequently, finding the cut-point MPV value to predict mortality was inconceivable. This was supposed to be from different MPV measurement methods; four of the ten studies used the electrical impedance method, three of the ten studies used the optical mean method, and another three were not mentioned. The difference in the methods of measurement affected the MPV values [33]. The use of different anticoagulants also affected the MPV values [34, 35]; only 5 of the 11 publications reported using EDTA as the anticoagulant $[17,18,20,24,26]$. The time from venipuncture to measurement could also have affected the MPV value; platelets become larger over time after obtaining blood [36]. EDTA can cause the swelling of platelets, so the analysis should be done within 1 hour when EDTA is 
TABLE 3: Sensitivity analysis of possible heterogeneity studies.

\begin{tabular}{|c|c|c|c|c|c|c|}
\hline Reason of exclusion & Excluded studies & Number of studies & $I$ square $(\%)$ & SMD & (95\% confidence interval) & $p$ value \\
\hline Lowest mortality & Kitazawa et al. [20] & 10 & 83.5 & 0.197 & $(-0.020$ to 0.414$)$ & 0.076 \\
\hline Highest mortality & Gao et al. [22] & 10 & 82.6 & 0.114 & $(-0.098$ to 0.326$)$ & 0.293 \\
\hline Lowest and highest mortality & $\begin{array}{c}\text { Kitazawa et al. [20] } \\
\text { Gao et al. [22] }\end{array}$ & 9 & 83.4 & 0.139 & $(-0.084$ to 0.361$)$ & 0.221 \\
\hline Small studies $(N<100)$ & $\begin{array}{l}\text { Becchi et al. [18] } \\
\text { Zampieri et al. [24] }\end{array}$ & 9 & 83.5 & 0.241 & $(0.023$ to 0.030$)$ & 0.030 \\
\hline
\end{tabular}

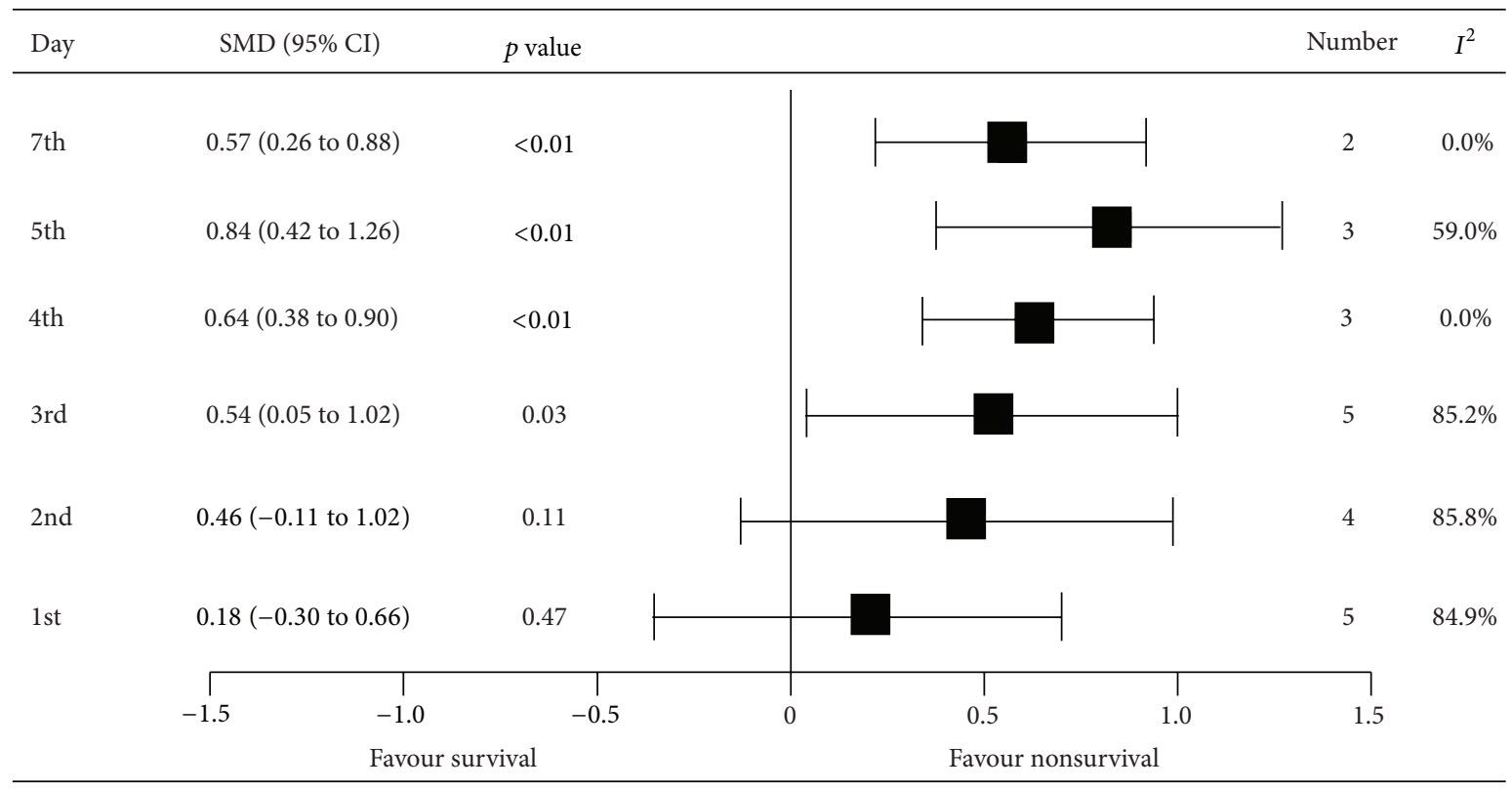

FIGURE 3: The summary of pooled mean differences of mean platelet volume on the observed day after admission between nonsurvivors and survivors.

used [34]. There were many conditions that might have influenced MPV such as antiplatelet condition, smoking, preexisting hematologic diseases, liver diseases, malignancy, various inflammatory diseases, or any recent transfusions [37-45]. The differences in the exclusion criteria in each study could also have caused some degree of heterogeneity. Nevertheless, such heterogeneity among critically ill patients is always found in real world clinical practice.

\section{Conclusions}

Initial high MPV might not be used as a prognostic marker of mortality in critically ill patients; however, subsequent MPV might be useful. Future studies with well-designed prospective cohort are warranted.

\section{Conflict of Interests}

The authors declare that there is no conflict of interests in this study.

\section{Acknowledgment}

The authors would like to thank Mrs. Chureerat Haiasch (medical technologist in clinical microscopy and diagnostic laboratory) who advised them on the details of the mean platelet volume measurement method in the laboratory processes.

\section{References}

[1] R. P. Dellinger, M. M. Levy, A. Rhodes, D. Annane, H. Gerlach, S. M. Opal et al., "Surviving sepsis campaign: international guidelines for management of severe sepsis and septic shock: 2012," Critical Care Medicine, vol. 41, no. 2, pp. 580-637, 2014.

[2] M. M. Levy, M. P. Fink, J. C. Marshall et al., “2001 SCCM/ESICM/ACCP/ATS/SIS international sepsis definitions conference," Intensive Care Medicine, vol. 29, no. 4, pp. 530-538, 2003.

[3] J.-L. Vincent, R. Moreno, J. Takala et al., "The SOFA (SepsisRelated Organ Failure Assessment) score to describe organ 
dysfunction/failure. On behalf of the Working Group on SepsisRelated Problems of the European Society of Intensive Care Medicine," Intensive Care Medicine, vol. 22, no. 7, pp. 707-710, 1996.

[4] J. C. Marshall, D. J. Cook, N. V. Christou, G. R. Bernard, C. L. Sprung, and W. J. Sibbald, "Multiple organ dysfunction score: a reliable descriptor of a complex clinical outcome," Critical Care Medicine, vol. 23, no. 10, pp. 1638-1652, 1995.

[5] J. R. Le Gall, J. Klar, S. Lemeshow et al., "The Logistic Organ Dysfunction system. A new way to assess organ dysfunction in the intensive care unit. ICU Scoring Group," The Journal of the American Medical Association, vol. 276, no. 10, pp. 802-810, 1996.

[6] C. B. Thompson, J. A. Jakubowski, P. G. Quinn, D. Deykin, and C. R. Valeri, "Platelet size as a determinant of platelet function," The Journal of Laboratory and Clinical Medicine, vol. 101, no. 2, pp. 205-213, 1983.

[7] C. B. Thompson, K. A. Eaton, S. M. Princiotta, C. A. Rushin, and C. R. Valeri, "Size dependent platelet subpopulations: relationship of platelet volume to ultrastructure, enzymatic activity, and function," British Journal of Haematology, vol. 50, no. 3, pp. 509-519, 1982.

[8] S. G. Chu, R. C. Becker, P. B. Berger et al., "Mean platelet volume as a predictor of cardiovascular risk: a systematic review and meta-analysis," Journal of Thrombosis and Haemostasis, vol. 8, no. 1, pp. 148-156, 2010.

[9] N. Sansanayudh, T. Anothaisintawee, D. Muntham, M. McEvoy, J. Attia, and A. Thakkinstian, "Mean platelet volume and coronary artery disease: a systematic review and meta-analysis," International Journal of Cardiology, vol. 175, no. 3, pp. 433-440, 2014.

[10] J. C. Arévalo-Lorido, J. Carretero-Gómez, A. Álvarez-Oliva, C. Gutiérrez-Montaño, J. M. Fernández-Recio, and F. NajarroDíez, "Mean platelet volume in acute phase of ischemic stroke, as predictor of mortality and functional outcome after 1 year," Journal of Stroke and Cerebrovascular Diseases, vol. 22, no. 4, pp. 297-303, 2013.

[11] S. Greisenegger, G. Endler, K. Hsieh, S. Tentschert, C. Mannhalter, and W. Lalouschek, "Is elevated mean platelet volume associated with a worse outcome in patients with acute ischemic cerebrovascular events?" Stroke, vol. 35, no. 7, pp. 1688-1691, 2004.

[12] X. Wan, W. Wang, J. Liu, and T. Tong, "Estimating the sample mean and standard deviation from the sample size, median, range and/or interquartile range," BMC Medical Research Methodology, vol. 14, article 135, 2014.

[13] G. A. Wells, B. Shea, D. O'Connell et al., The Newcastle-Ottawa Scale (NOS) for Assessing the Quality of Nonrandomized Studies in Meta-Analyses, The Ottawa Hospital Foundation, Ontario, Canada, 2014, http://www.ohri.ca/programs/clinical_epidemiology/oxford.asp.

[14] J. Lau, J. P. A. Ioannidis, and C. H. Schmid, "Quantitative synthesis in systematic reviews," Annals of Internal Medicine, vol. 127, no. 9, pp. 820-826, 1997.

[15] J. P. T. Higgins and S. G. Thompson, "Quantifying heterogeneity in a meta-analysis," Statistics in Medicine, vol. 21, no. 11, pp. 15391558, 2002.

[16] M. Egger, G. D. Smith, M. Schneider, and C. Minder, "Bias in meta-analysis detected by a simple, graphical test," The British Medical Journal, vol. 315, no. 7109, pp. 629-634, 1997.

[17] Y. Kucukardali, Y. Onem, H. Terekeci, F. Tangi, B. Sahan, A. A. Erikci et al., "Mean Platelet Volume (MPV) in Intensive
Care Unit (ICU) Patients: is it a useful parameter in assessing prediction for mortality?" Journal of Medicine and Medical Sciences, vol. 1, no. 3, pp. 61-64, 2010.

[18] C. Becchi, M. Al Malyan, L. P. Fabbri, M. Marsili, V. Boddi, and S. Boncinelli, "Mean platelet volume trend in sepsis: is it a useful parameter?" Minerva Anestesiologica, vol. 72, no. 9, pp. 749-756, 2006.

[19] E. Guclu, Y. Durmaz, and O. Karabay, "Effect of severe sepsis on platelet count and their indices," African Health Sciences, vol. 13, no. 2, pp. 333-338, 2013.

[20] T. Kitazawa, Y. Yoshino, K. Tatsuno, Y. Ota, and H. Yotsuyanagi, "Changes in the mean platelet volume levels after bloodstream infection have prognostic value," Internal Medicine, vol. 52, no. 13, pp. 1487-1493, 2013.

[21] F. Sadaka, P. L. Donnelly, M. T. Griffin, J. O'Brien, and R. Lakshmanan, "Mean platelet volume is not a useful predictor of mortality in septic shock," Journal Blood Disorders and Transfusion, vol. 5, no. 2, p. 194, 2014.

[22] Y. Gao, Y. Li, X. Yu et al., "The impact of various platelet indices as prognostic markers of septic shock," PLoS ONE, vol. 9, no. 8, Article ID e103761, 2014.

[23] C. Sezgi, M. Taylan, H. Kaya et al., "Alterations in platelet count and mean platelet volume as predictors of patient outcome in the respiratory intensive care unit," The Clinical Respiratory Journal, vol. 9, no. 4, pp. 403-408, 2015.

[24] F. G. Zampieri, O. T. Ranzani, V. Sabatoski et al., "An increase in mean platelet volume after admission is associated with higher mortality in critically ill patients," Annals of Intensive Care, vol. 4, article 20, 2014.

[25] Z. Zhang, X. Xu, H. Ni, and H. Deng, "Platelet indices are novel predictors of hospital mortality in intensive care unit patients," Journal of Critical Care, vol. 29, no. 5, pp. 885.el-885.e6, 2014.

[26] C. H. Kim, S. J. Kim, M. J. Lee et al., "An increase in mean platelet volume from baseline is associated with mortality in patients with severe sepsis or septic shock," PLoS ONE, vol. 10, no. 3, Article ID e0119437, 2015.

[27] S. Zhang, Y. L. Cui, M. Y. Diao, D. C. Chen, and Z. F. Lin, "Use of platelet indices for determining illness severity and predicting prognosis in critically ill patients," Chinese Medical Journal, vol. 128, no. 15, pp. 2012-2018, 2015.

[28] A. Eberhardt, F. Lessig, K. Schreiter et al., "Mean platelet volume (MPV) is an outcome marker in sepsis patients," International Journal of Infectious Diseases, vol. 16, supplement 1, p. e218, 2012.

[29] G. Davì and C. Patrono, "Platelet activation and atherothrombosis," The New England Journal of Medicine, vol. 357, no. 24, pp. 2482-2494, 2007.

[30] A. Zarbock, R. K. Polanowska-Grabowska, and K. Ley, "Plateletneutrophil-interactions: linking hemostasis and inflammation," Blood Reviews, vol. 21, no. 2, pp. 99-111, 2007.

[31] C. B. Thompson, J. A. Jakubowski, P. G. Quinn, D. Deykin, and C. R. Valeri, "Platelet size and age determine platelet function independently," Blood, vol. 63, no. 6, pp. 1372-1375, 1984.

[32] J. Van der Lelie and A. E. G. K. Von dem Borne, "Increased mean platelet volume in septicaemia," Journal of Clinical Pathology, vol. 36, no. 6, pp. 693-696, 1983.

[33] V. Latger-Cannard, M. Hoarau, S. Salignac, D. Baumgart, P. Nurden, and T. Lecompte, "Mean platelet volume: comparison of three analysers towards standardization of platelet morphological phenotype," International Journal of Laboratory Hematology, vol. 34, no. 3, pp. 300-310, 2012. 
[34] M. S. Dastjerdi, T. Emami, A. Najafian, and M. Amini, "Mean platelet volume measurement, EDTA or citrate?" Hematology, vol. 11, no. 5-6, pp. 317-319, 2006.

[35] M. D. Lancé, R. van Oerle, Y. M. C. Henskens, and M. A. E. Marcus, "Do we need time adjusted mean platelet volume measurements?" Laboratory Hematology, vol. 16, no. 3, pp. 2831, 2010.

[36] S. R. Jackson and J. M. Carter, "Platelet volume: laboratory measurement and clinical application," Blood Reviews, vol. 7, no. 2, pp. 104-113, 1993.

[37] W. S. Lee and T.-Y. Kim, "Alcoholic fatty liver disease and alcoholic liver cirrhosis may be differentiated with mean platelet volume and platelet distribution width," Platelets, vol. 21, no. 7, pp. 584-585, 2010.

[38] K. Kario, T. Matsuo, and K. Nakao, "Cigarette smoking increases the mean platelet volume in elderly patients with risk factors for atherosclerosis," Clinical and Laboratory Haematology, vol. 14, no. 4, pp. 281-287, 1992.

[39] S. Yazici, M. Yazici, B. Erer et al., "The platelet indices in patients with rheumatoid arthritis: mean platelet volume reflects disease activity," Platelets, vol. 21, no. 2, pp. 122-125, 2010.

[40] S. Yazici, M. Yazici, B. Erer et al., "The platelet functions in patients with ankylosing spondylitis: anti-TNF- $\alpha$ therapy decreases the mean platelet volume and platelet mass," Platelets, vol. 21, no. 2, pp. 126-131, 2010.

[41] H. Kayahan, M. Akarsu, M. A. Ozcan et al., "Reticulated platelet levels in patients with ulcerative colitis," International Journal of Colorectal Disease, vol. 22, no. 12, pp. 1429-1435, 2007.

[42] A. Y. Gasparyan, A. Stavropoulos-Kalinoglou, T. E. Toms, K. M. J. Douglas, and G. D. Kitas, "Association of mean platelet volume with hypertension in rheumatoid arthritis," Inflammation \& Allergy-Drug Targets, vol. 9, no. 1, pp. 45-50, 2010.

[43] S. Guthikonda, C. L. Alviar, M. Vaduganathan et al., "Role of reticulated platelets and platelet size heterogeneity on platelet activity after dual antiplatelet therapy with aspirin and clopidogrel in patients with stable coronary artery disease," Journal of the American College of Cardiology, vol. 52, no. 9, pp. 743-749, 2008.

[44] Y. G. Kim, J.-W. Suh, C.-H. Yoon et al., "Platelet volume indices are associated with high residual platelet reactivity after antiplatelet therapy in patients undergoing percutaneous coronary intervention," Journal of Atherosclerosis and Thrombosis, vol. 21, no. 5, pp. 445-453, 2014.

[45] J.-Y. Li, Y. Li, Z. Jiang, R.-T. Wang, and X.-S. Wang, "Elevated mean platelet volume is associated with presence of colon cancer," Asian Pacific Journal of Cancer Prevention, vol. 15, no. 23, pp. 10501-10504, 2014. 


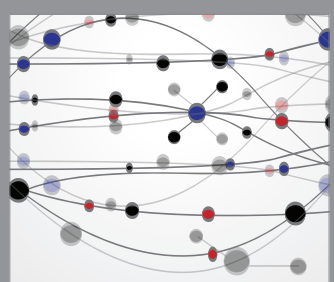

The Scientific World Journal
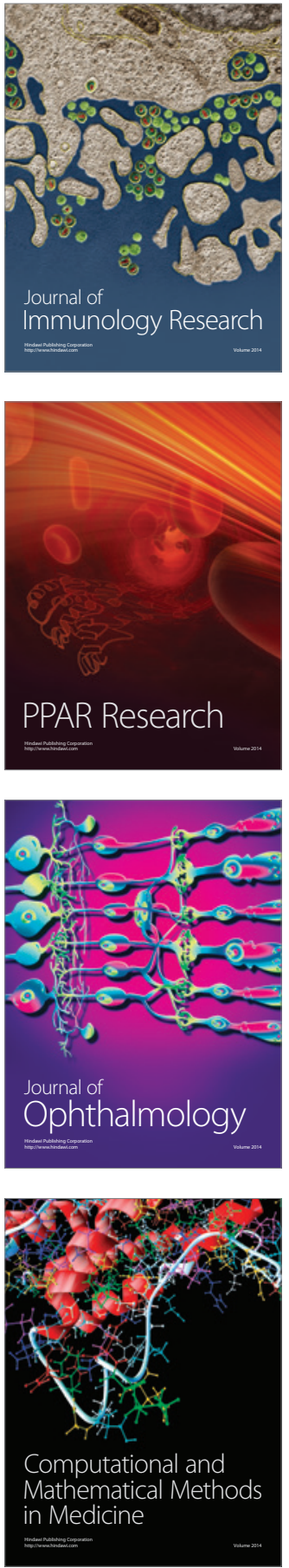

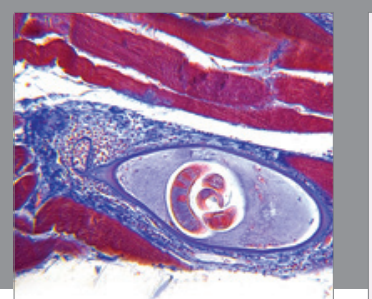

Gastroenterology Research and Practice

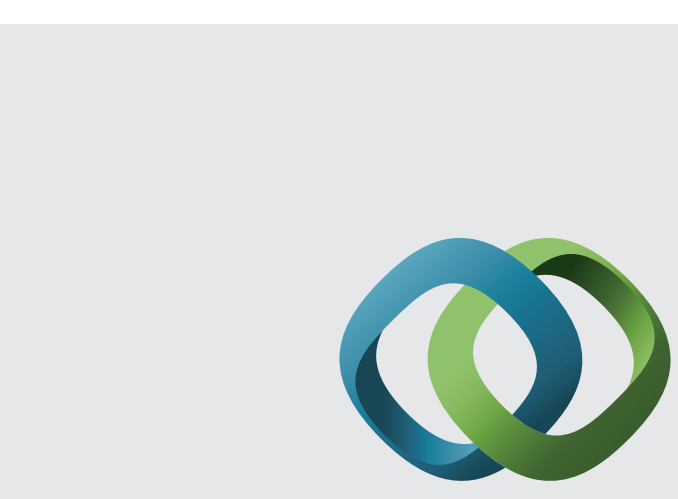

\section{Hindawi}

Submit your manuscripts at

http://www.hindawi.com
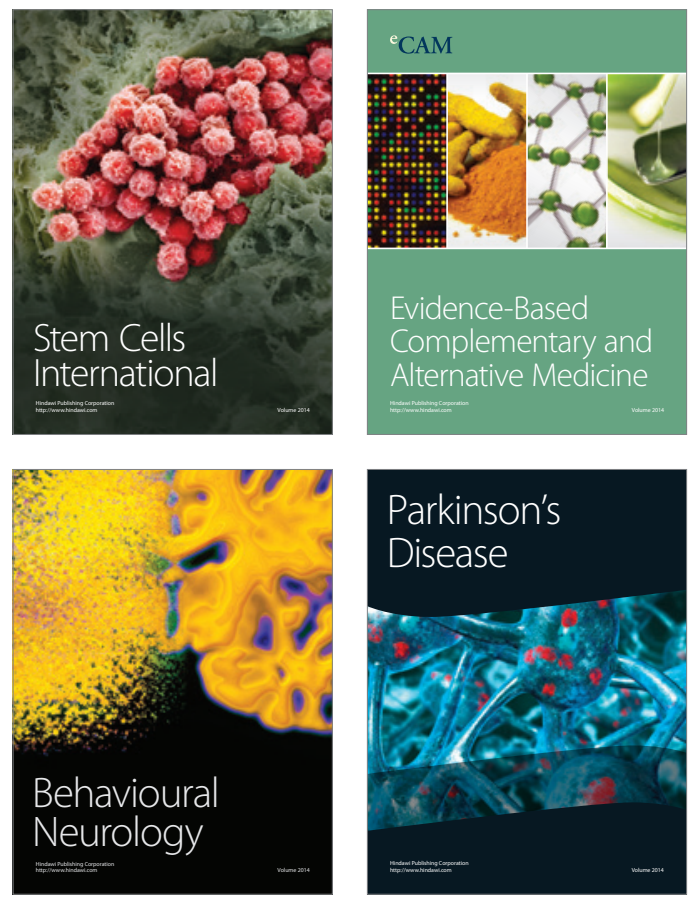
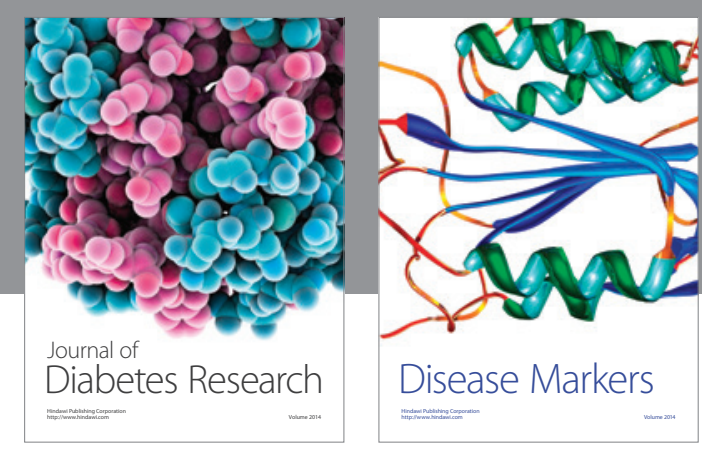

Disease Markers
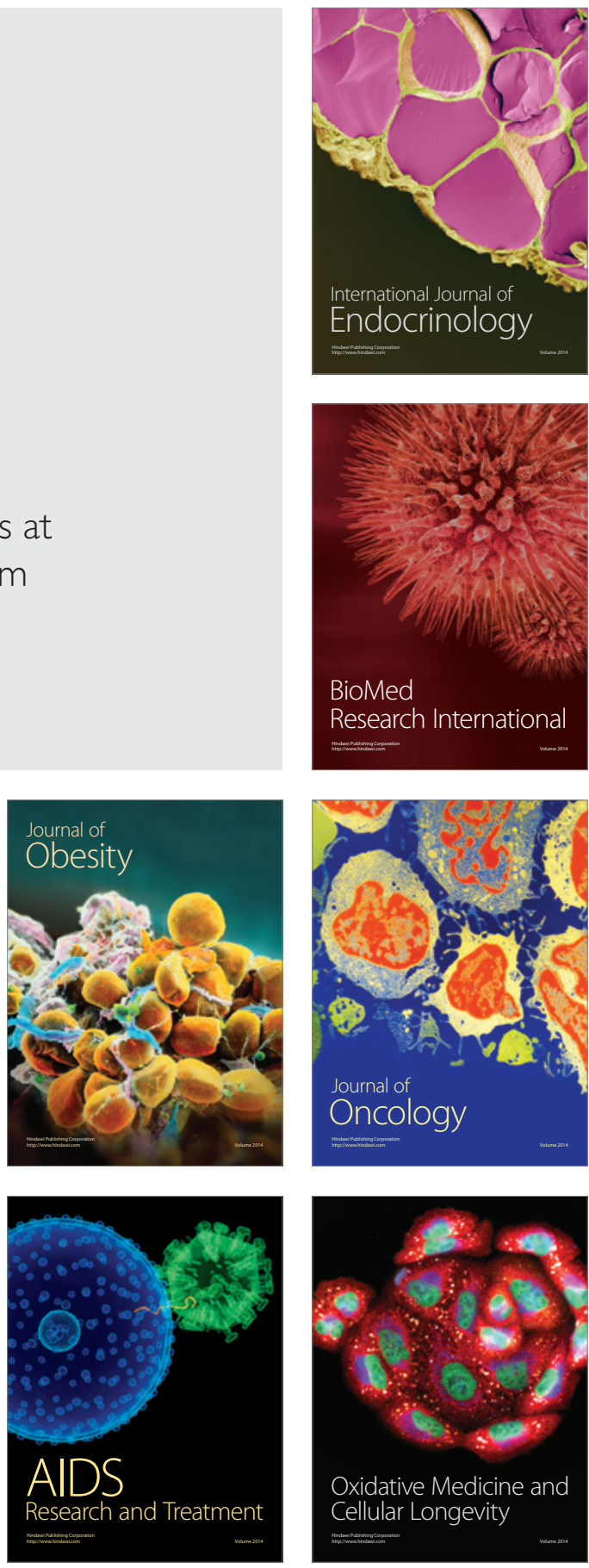